\title{
Activity and functional interaction of alternative oxidase and uncoupling protein in mitochondria from tomato fruit
}

F.E. Sluse ${ }^{1}$ and W. Jarmuszkiewicz ${ }^{2}$
${ }^{1}$ Laboratory of Bioenergetics, Centre of O xygen, Research, D evelopment, Institute of Chemistry B6, U niversity of Liege, Liege, Belgium

2Department of Bioenergetics, Adam Mickiewicz University, Poznan, Poland

\section{Correspondence \\ F.E. Sluse \\ Laboratory of Bioenergetics \\ Centre of $O$ xygen, Research, \\ Development \\ Institute of Chemistry B6 \\ University of Liege \\ Sart-Tilman \\ B-4000 Liege \\ Belgium \\ Fax: + 32-4-366-2878 \\ E-mail: F.Sluse@ulg.ac.be \\ Presented at the XXVIII Annual Meeting of the Brazilian Society of Biochemistry and Molecular Biology, Caxambu, MG, Brasil, May 22-25, 1999. \\ Research supported by the Belgian "Fonds de la Recherche Fondamentale Collective", the Polish- Belgian Joint Research Program, and by FAPESP, CN Pq, PADCT and PRONEX}

Received O ctober 25, 1999 Accepted November 29, 1999

\section{Abstract}

Cyanide-resistant alternative oxidase (AOX) is not limited to plant mitochondria and is widespread among several types of protists. The uncoupling protein (UCP) is much more widespread than previously believed, not only in tissues of higher animals but also in plants and in an amoeboid protozoan. The redox energy-dissipating pathway (AOX) and the proton electrochemical gradient energy-dissipating pathway (UCP) lead to the same final effect, i.e., a decrease in ATP synthesis and an increase in heat production. Studies with green tomato fruit mitochondria show that both proteins are present simultaneously in the membrane. This raises the question of a specific physiological role for each energy-dissipating system and of a possible functional connection between them (shared regulation). Linoleic acid, an abundant free fatty acid in plants which activates UCP, strongly inhibits cyanide-resistant respiration mediated by AOX. Moreover, studies of the evolution of AOX and UCP protein expression and of their activities during post-harvest ripening of tomato fruit show that $\mathrm{AOX}$ and plant UCP work sequentially: AOX activity decreases in early post-growing stages and UCP activity is decreased in late ripening stages. Electron partitioning between the alternative oxidase and the cytochrome pathway as well as $\mathrm{H}^{+}$gradient partitioning between ATP synthase and UCP can be evaluated by the ADP/O method. This method facilitates description of the kinetics of energy-dissipating pathways and of ATP synthase when state 3 respiration is decreased by limitation of oxidizable substrate.

\section{Introduction}

The respiratory chain of plant mitochondria contains three redox energy-dissipating steps that, in contrast to complexes I, III, and IV, do not build a proton electrochemical gradient. An external NAD $(\mathrm{P}) \mathrm{H}$ dehydrogenase and an internal NAD(P)H dehydrogenase, both insensitive to rotenone, reduce

\section{Key words}

- Plant mitochondria

- Alternative oxidase

- Uncoupling protein

- Regulation

- Electron partitioning ubiquinone. An alternative oxidase (AOX), insensitive to cyanide, oxidizes ubiquinol and reduces $\mathrm{O}_{2}$ (for an overview, see 1 and for recent comprehensive reviews, see 2,3). If complex I is inactivated by rotenone and if the cytochrome pathway is blocked by cyanide, electron donors such as NADH and succinate can reduce oxygen without energy conservation, producing only heat 
from redox energy.

In addition to these redox energy-dissipating processes, some plant mitochondria contain another energy-dissipating system, a plant uncoupling mitochondrial protein named PUMP which dissipates the $\mathrm{H}^{+}$gradient $(4,5)$. This protein has been shown (6-8) to work in a manner similar to the mammalian uncoupling protein (UCP) $(9,10)$ as a mitochondrial carrier that probably exports anionic free fatty acid (FFA) across the inner mitochondrial membrane. Protonated fatty acids re-enter into the mitochondrial matrix by diffusion. By removing the anionic FFA from the matrix back into the intermembrane space, PUMP enables $\mathrm{H}^{+}$re-entry through a fatty acid cycling process, bypassing the ATP synthase route and consequently uncoupling respiration from phosphorylation and dissipating the proton electrical-energy gradient into heat.

The aim of this paper is to show that two energy-dissipating systems, AOX and PUMP, are present together in green tomato mitochondria, to describe the functional connection between them, to evaluate the evolution of the AOX and PUMP proteins and activities during post-harvest ripening of tomato fruit, and to examine the kinetics of the contributions of AOX, PUMP, and ATP synthase to overall state 3 respiration.

\section{Role of the two energy-dissipating systems}

The two energy-dissipating systems, AOX and PUMP, lead to the same final effect, i.e.,

Figure 1 - Energy balance of the cell. Proposed roles of alternative oxidase (AOX) and plant uncoupling mitochondrial protein (PUMP) in energy imbalance of cell metabolism (see text).

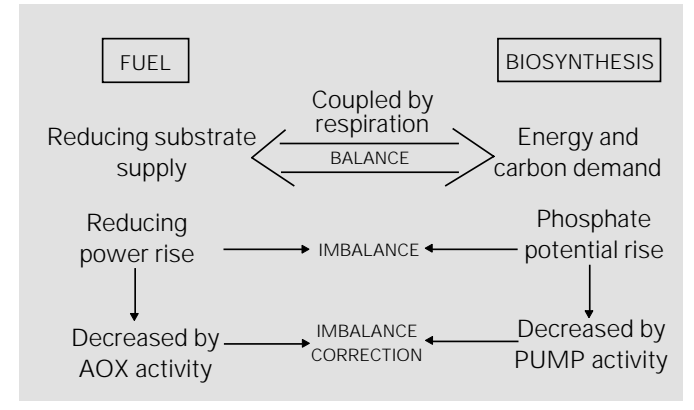

a decrease in ATP synthesis and an increase in heat production. Thus they may play a role in thermogenesis, as shown for alternative oxidase in plant thermogenic tissues where its activity leads to a temperature rise involved in reproductive processes $(11,12)$. An increase in temperature is also observed during the ripening of climacteric fruits and has been attributed to the stimulation of AOX activity (13-15).

However, in non-thermogenic tissues, PUMP and AOX may play a more fundamental role at the level of the energy balance of the cell. Indeed, the supply of reducing substrates and the energy and carbon demand for biosynthesis are coupled by respiration. Metabolic conditions that lead to a high reducing power and a high phosphate potential reflect an imbalance between the supply and demand processes. An increase in AOX activity, which is not directly controlled by the energy status of the cell, will decrease the reducing power. On the other hand, an increase in PUMP activity, which consumes the $\mathrm{H}^{+}$electrochemical gradient, will decrease the phosphate potential. Thus, both activities could correct the imbalance (Figure 1).

Therefore, a possible connection between the activities of AOX and PUMP through a shared regulation and their possible complementarity could be of the utmost importance for the efficiency of oxidative phosphorylation and for the energy status of the plant cell. Such a connection could shed light on the reasons for the coexistence of the two energy-dissipating systems in plants.

\section{Specific regulation of $A O X$ and PUMP}

The AOX activity is finely tuned by several parameters. Regulation of AOX can occur i) at the level of gene expression that modulates the amount of protein in the membrane, ii) by post-translational modification that modifies the redox status of the enzyme 
and its activity, and iii) by chemical modification (formation of thiohemiacetal from pyruvate) that activates AOX. AOX activity also depends on the substrate availability, i.e., the total concentration of ubiquinone in the membrane, its redox state and $\mathrm{O}_{2}$ concentration (1-3).

The PUMP activity is also regulated at different levels such as gene expression, allosteric inhibition (GTP, ATP), and substrate availability (concentration of anionic FFA on both sides of the membrane). Bovine serum albumin (BSA, free of fatty acids) inhibits PUMP activity by chelating FFA. Moreover, the transmembrane electrical potential (negative inside) and $\mathrm{pH}$ difference (acidic outside) are the driving forces of the fatty acid cycling $\mathrm{H}^{+}$re-uptake (4-8).

\section{Respiratory network in tomato mitochondria}

Elements of the plant mitochondrial respiratory network investigated in green tomato fruit with succinate as oxidizable substrate (+ rotenone to block complex I) are shown in Figure 2. Two pathways, the cytochrome pathway and AOX, transfer electrons from succinate to oxygen. Three pathways, ATP synthesis, PUMP activity, and $\mathrm{H}^{+}$ leakage, consume the $\mathrm{H}^{+}$electrochemical gradient built up by the cytochrome pathway. Except for $\mathrm{H}^{+}$leakage, each pathway may be blocked by specific inhibitors such as cyanide, benzohydroxamic acid (BHAM), oligomycin, GTP or BSA.

\section{AOX-PUMP connection}

Since the common final effect of the AOX and PUMP activities is a decrease in ATP synthesis efficiency, it can be quantified by $\mathrm{ADP} / \mathrm{O}$ ratio measurements (16). The effect of various respiratory conditions on this ratio is shown in Table 1. The control value of $\mathrm{ADP} / \mathrm{O}$ with succinate, equal to 1.43, is obtained when both AOX and PUMP are blocked. When AOX is activated by dithiothreitol and pyruvate and PUMP is blocked, the ratio decreases to 1.29 . When PUMP is activated and AOX blocked the ratio decreases even more to 1.01. But when both energy-dissipating systems are activated together, no further significant decrease is observed compared to the situation of PUMP activation and AOX blockade. This puzzling observation is the first indication that the AOX and PUMP activities are somehow connected as they have no cumulative effect on $\mathrm{ADP} / \mathrm{O}$.

An explanation was obtained from an experiment where both cyanide-resistant res-

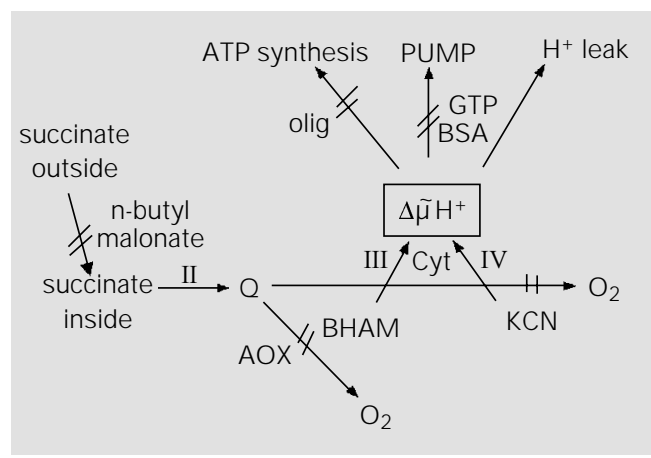

Table 1 - Effect of various incubation conditions on the ADP/O ratio.

ADP/O is measured during an ADP pulse (0.17 $\mathrm{mM})$ with succinate $(10 \mathrm{mM})$ plus rotenone as oxidizable substrate. $\mathrm{O}$ is the total amount of oxygen consumed during state 3 respiration. The concentrations used were $2 \mathrm{mM}$ benzohydroxamic acid (BHAM), 0.5\% bovine serum albumin (BSA), $1 \mathrm{mM}$ guanosine triphosphate (GTP), $1 \mathrm{mM}$ dithiothreitol (DTT), and $0.15 \mathrm{mM}$ pyruvate (Pyr). First line: control conditions, AOX and PUMP inhibited. Second line: AOX activated, PUMP inhibited. Third line: AOX inhibited, PUMP activated. Fourth line: AOX and PUMP activated. For details see Ref. 16. LA, Linoleic acid.

\begin{tabular}{|c|c|}
\hline Conditions & ADP/O \\
\hline$+\mathrm{GTP}+\mathrm{BSA}+\mathrm{BHAM}$ & $1.43 \pm 0.03$ \\
\hline$+\mathrm{GTP}+\mathrm{BSA}+\mathrm{DTT}+\mathrm{Pyr}$ & $1.29 \pm 0.10$ \\
\hline$+3.9 \mu \mathrm{M}$ LA+BHAM & $1.01 \pm 0.03$ \\
\hline$+3.9 \mu \mathrm{M}$ LA+DTT+Pyr & $0.95 \pm 0.13$ \\
\hline
\end{tabular}

Figure 2 - Respiratory network in tomato mitochondria. Complex II, Succinate dehydrogenase; complex III, cytochrome $\mathrm{bc}_{1}$; complex IV, cytochrome oxidase; AOX, alternative oxidase; Cyt, cytochrome pathway; Q, ubiquinone; PUMP, plant uncoupling mitochondrial protein; $\Delta \widetilde{\mu} \mathrm{H}^{+}$, proton electrochemical gradient built up by the cytochrome pathway; BHAM, benzohydroxamic acid; KCN, potassium cyanide; olig, oligomycin; GTP, guanosine triphosphate; BSA, bovine serum albumin; n-butyl malonate, inhibitor of succinate uptake. 
piratory rate and linoleic acid (LA)-induced respiratory rate were measured at increasing LA concentrations with green tomato fruit mitochondria fully depleted of endogenous FFA (Figure 3). In the presence of BHAM and oligomycin, the LA-induced respiration, which reflects PUMP activity, increases with increasing LA concentrations and $50 \%$ maximal stimulation is reached at $10 \mu \mathrm{M}$ LA (Figure 3, circles). The cyanide-resistant AOX-mediated respiration decreases with increasing LA concentrations and 50\% inhibition was reached at less than $4 \mu \mathrm{M}$ LA (Figure 3, squares). AOX activity may be considered to be blocked at $15 \mu \mathrm{M}$ LA. This experiment demonstrates for the first time how an increase in FFA level affects both energy-dissipating systems, but in opposite directions. Moreover, it seems that AOX activity is more sensitive to the inhibitory effect of LA than PUMP activity is sensitive to the activation by LA. The inhibitory effect of LA on cyanide-resistant respiration was also shown in mitochondria of Arum maculatum (17) and Hansenula anomala (18).

The results described above show how the activity of AOX can be progressively switched off by an increase in FFA level in plant cells. They also show that AOX and PUMP never seem to work simultaneously at least at their maximal activity, and it is likely that when PUMP reaches high activity AOX is already fully switched off (16).

Figure 3 - Cyanide-resistant respiration and linoleic acid (LA)-induced respiration versus LA concentration. Cyanide-resistant respiration (squares) $(+1 \mathrm{mM}$ $\mathrm{KCN}$ ) was measured in the presence of dithiothreitol (DTT) plus pyruvate. LA-induced respiration (circles) was measured in the presence of $2 \mathrm{mM}$ benzohydroxamic acid (BHAM). Fully FFA-depleted green tomato mitochondria (16) were incubated with 10 $\mathrm{mM}$ succinate, $5 \mu \mathrm{M}$ rotenone, $2.5 \mu \mathrm{g}$ oligomycin/mg protein, and $0.17 \mathrm{mM}$ ATP.

\section{AOX and PUMP activity during tomato fruit post-harvest ripening}

If AOX and PUMP are not working together they could work sequentially during the life of plant cell according to its particular physiological state. Ripening of fruits may provide an interesting model to study this eventuality. Indeed, thermogenesis occurs during fruit ripening (13-15) and FFA concentration increases during the post-growing stage $(19,20)$.

The evolution of cyanide-resistant respiration, ATP synthesis-sustained respiration and PUMP-sustained respiration can be measured in isolated mitochondria from tomato fruit during post-harvest ripening and the alterations in the amount of AOX and PUMP proteins by immunological detection can also be assessed throughout the period of ripening (21).

ATP synthesis-sustained respiration was measured in state 3 in the presence of succinate (+ rotenone) as oxidizable substrate, BHAM and GTP, and BSA as inhibitors of AOX and PUMP, respectively (Figure 4B). It represents the activity of a network pathway involving respiratory complexes II, III, IV and ATP synthase and $\mathrm{H}^{+}$leak in state 3 (see Figure 2). State 3 was initiated in the presence of GTP and BSA, followed by the addition of BHAM and terminated by $\mathrm{KCN}$ addition. The difference between respiratory rate with BHAM and respiratory rate with BHAM and KCN gives the ATP synthesis-sustained respiration. When plotted against the stage of ripeness, it decreases up to the orange stage. This decline subsequently stabilizes between the orange and red stages (Figure 4B, ATP synthesis).

AOX-(cyanide-resistant)-sustained respiration measured in state 3 in the presence of $\mathrm{KCN}$, GTP, and BSA (Figure 4B) corresponds to the activity of the electron transport pathway including complex II and AOX (see Figure 2). State 3 was initiated in the presence of GTP and BSA followed by the 
addition of $\mathrm{KCN}$ and terminated by BHAM. The difference between respiratory rate with $\mathrm{KCN}$ and respiratory rate with $\mathrm{KCN}$ and BHAM (i.e., residual respiration) gives the AOX-sustained respiration. When plotted against the stage of ripeness its activity decreases from the green to the orange stage and then stabilizes (Figure 4B, AOX).

PUMP-sustained respiration was measured in state 4 in the presence of oligomycin and BHAM after the addition of $10 \mu \mathrm{M}$ LA (Figure 4B). The respiratory rate in the presence of LA represents the activity of the respiratory pathway that includes complexes II, III, IV, PUMP, and $\mathrm{H}^{+}$leak (see Figure 2). State 4 was initiated in the presence of oligomycin and BHAM, followed by the addition of $10 \mu \mathrm{M} \mathrm{LA}$ and terminated by BSA plus GTP. The respiratory rate after LA addition gives PUMP-sustained respiration plus $\mathrm{H}^{+}$ leak in the presence of $10 \mu \mathrm{MLA}$ (negligible due to an LA-induced drop in state 4 membrane potential). The respiratory rate after BSA plus GTP gives the proton leakage in the absence of LA. With the stage of ripeness the total PUMP-sustained respiration plus the $\mathrm{H}^{+}$leak markedly decreased between the yellow and orange stages and then stabilized (Figure 4B, PUMP). Proton leak in state $4(+$ BSA, GTP) did not change significantly with ripening (data not shown).

Immunoblotting of mitochondrial proteins of tomato fruit allowed the detection of AOX and PUMP, clearly indicating that both proteins are present simultaneously in green tomato fruit mitochondria (Figure 4A). The level of AOX protein decreases with ripening from green stage forward, and parallels the decrease in AOX-sustained respiration. Changes in PUMP protein levels with the stage of ripeness were less pronounced, and a decrease occurred after the yellow stage, as was also the case for the PUMP-sustained respiration.

These results indicate a clear regulation of AOX activity through a decrease in protein expression during tomato fruit ripening, and a decrease in PUMP protein expression only after the yellow stage that parallels PUMP activity. These results suggest that AOX and PUMP work sequentially. AOX would be active mainly during the growing period thereby providing a safety balance between redox potential, phosphate potential, and biosynthesis demand, whereas PUMP would start working in the post-growing stage when the FFA concentration increases, thereby providing a mechanism for
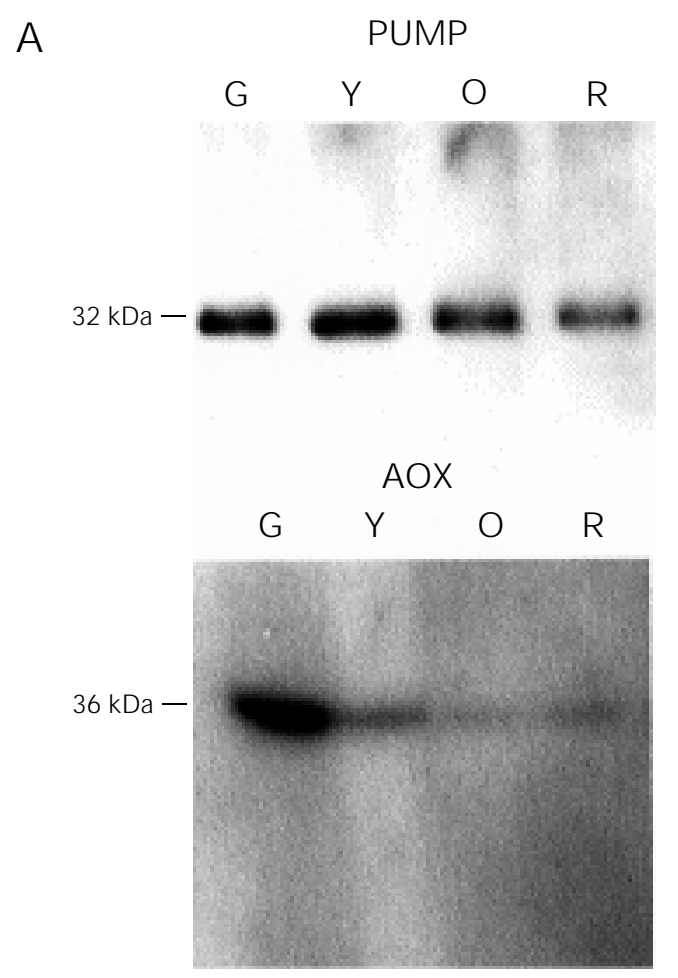

B

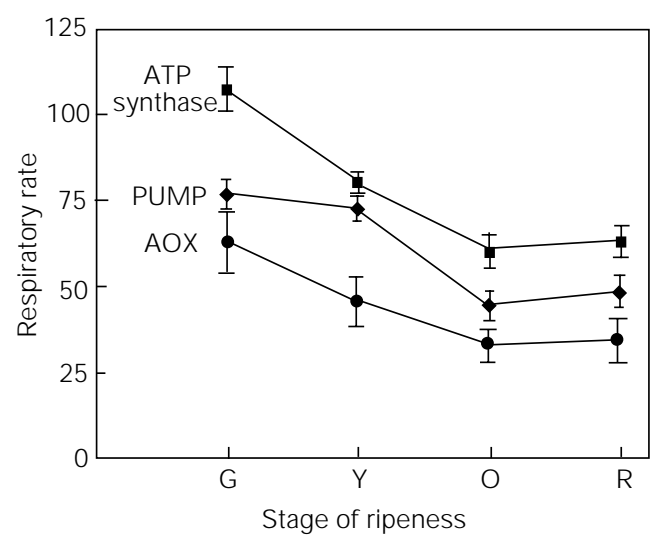

Figure 4 - Immunodetection of alternative oxidase (AOX) and plant uncoupling mitochondrial protein (PUMP) and analysis of respiratory activities during ripening. A, Immunoblot analysis of tomato mitochondrial proteins at different stages of ripeness (G, green; Y, yellow; O, orange, and $\mathrm{R}$, red). Monoclonal antibodies against the Sauromatum guttatum AOX and polyclonal antibodies against the Solanum tuberosum PUMP were used (for details see 21). B, Respiratory rate sustained by ATP synthase activity (squares), by LAinduced PUMP activity (lozenges), and by AOX activity (circles) at different stages of ripeness $(G, Y, O, R)$ are measured as described in the text and in Ref. 21. 
heat generation via a decrease in the efficiency of oxidative phosphorylation in parallel with the termination of biosynthetic processes.

\section{True contributions of AOX, PUMP and ATP synthesis to state 3 respiration}

The respiratory activities described above (i.e., ATP synthesis-sustained respiration, AOX-sustained respiration, and PUMP-sustained respiration) are restricted to situations in which one of the activities is functioning while the other two are blocked. Therefore, these measurements do not reflect the true contributions of each of the three pathways to the overall state 3 respiration, as any change in one inevitably affects the others.

The operation of an energy-dissipating system decreases the ATP synthesis efficiency, so pair measurements of the ADP/O ratios in the presence or in the absence of a specific inhibitor of the energy-dissipating pathway allow calculation of the true contributions of both the energy-dissipating and energy-conserving pathways. The ADP/O method is valid if several requirements are fulfilled as described in Ref. 1. In order to describe how the contribution of each pathway changes with variation of state 3 respiratory rate, n-butyl malonate, a non-penetrating competitive inhibitor of succinate uptake, was used to decrease succinate availability and the rate of the quinone-reducing step (see Figure 2).

In the absence of FFA (no PUMP activity), the AOX contribution and the cytochrome pathway contribution to state 3 respiration can be determined $(1,22,23)$. Indeed, if $\mathrm{V}_{3}=\mathrm{V}_{\text {cyt }}+\mathrm{V}_{\mathrm{alt}}$, and if

$\alpha=\frac{A D P / O}{(A D P / O)_{\text {cyt }}}=\frac{\frac{A D P}{O_{\text {cyt }}+O_{\text {alt }}}}{\frac{A D P}{O_{\text {cyt }}}}=\frac{O_{\text {cyt }}}{O_{\text {cyt }}+O_{\text {alt }}}=\frac{\mathrm{V}_{\text {cyt }}}{\mathrm{V}_{\text {cyt }}+\mathrm{V}_{\text {alt }}}$

where $\mathrm{ADP} / \mathrm{O}$ is the $\mathrm{ADP} / \mathrm{O}$ ratio in the absence of BHAM (when both respiratory pathways are active), (ADP/O) $)_{\text {cyt }}$ is the ADP/ $\mathrm{O}$ ratio in the presence of BHAM (when only the cytochrome pathway is active), and $\mathrm{O}_{\text {cyt }}$ and $\mathrm{O}_{\text {alt }}$ are the amounts of oxygen taken up related to the activities of the cytochrome and alternative pathways, respectively. Then: $\mathrm{V}_{3} \times \alpha=\mathrm{V}_{\text {cyt }}=$ contribution of the cytochrome pathway, and $\mathrm{V}_{3}-\mathrm{V}_{\text {cyt }}=\mathrm{V}_{\text {alt }}=$ contribution of the alternative pathway.

In the absence of AOX activity (+ BHAM), the PUMP contribution and the energy conservative part of the cytochrome pathway, i.e., ATP synthesis contribution to state 3 respiration + BHAM can be calculated at a given LA concentration (8, 23). Indeed, if $\mathrm{V}_{3+\mathrm{LA}}=\mathrm{V}_{\text {cyt cons }}+\mathrm{V}_{\mathrm{PUMP}}$, and if

$$
\beta=\frac{(A D P / O)_{+L A}}{(A D P / O)_{-L A}}=\frac{\frac{A D P}{O_{\text {cons }}+O_{\text {diss }}}}{\frac{A D P}{O_{\text {cons }}}}=\frac{O_{\text {cons }}}{O_{\text {cons }}+O_{\text {diss }}}=\frac{V_{\text {cyt cons }}+V_{\text {PUMP }}}{V_{\text {cuns }}}
$$

where $(\mathrm{ADP} / \mathrm{O})_{+\mathrm{LA}}$ is the $\mathrm{ADP} / \mathrm{O}$ ratio in the presence of $\mathrm{LA}$ (when both conservative and dissipating paths are active), (ADP/O) $)_{-\mathrm{LA}}$ is the ADP/O ratio when PUMP is inactive, and $\mathrm{O}_{\text {cons }}$ and $\mathrm{O}_{\text {diss }}$ are the amounts of oxygen taken up related to ATP synthesis and PUMP activity, respectively. Then: $\mathrm{V}_{3+\mathrm{LA}} \times \mathrm{B}=$ $\mathrm{V}_{\text {cyt cons }}=$ contribution of ATP synthase to the cytochrome pathway activity, and $\mathrm{V}_{3+\mathrm{LA}}$ $\mathrm{V}_{\text {cyt cons }}=\mathrm{V}_{\text {PUMP }}=$ contribution of PUMP to the cytochrome pathway activity.

Practically, the method consists of measuring the $\mathrm{ADP} / \mathrm{O}$ ratios and the rate of state 3 respiration during an ADP pulse $(\mathrm{O}$ is the total amount of oxygen consumed during state 3).

Figure 5 shows the effect of $3.9 \mu \mathrm{M}$ LA on the $\mathrm{ADP} / \mathrm{O}$ ratio plotted against the reciprocal of state 3 respiration $\left(\mathrm{V}_{3}\right)$ which is varied with n-butyl malonate. In the presence of $\mathrm{LA}$, the $\mathrm{ADP} / \mathrm{O}$ ratio increases when $\mathrm{V}_{3}$ increases. In the absence of LA, the ADP/ $\mathrm{O}$ is constant when $\mathrm{V}_{3}$ is decreased. This constancy is one of the requirements for the validity of the ADP/O method. 
The contributions of ATP synthesis and PUMP activity to state 3 respiration were calculated from the pair measurements of $\mathrm{ADP} / \mathrm{O}$ and $\mathrm{V}_{3}$ (in the presence or absence of $3.9 \mu \mathrm{M} \mathrm{LA}$ ), and plotted against $\mathrm{V}_{3}$ (Figure 6). ATP synthesis contribution $\left(\mathrm{V}_{\text {cyt cons }}\right)$ to $\mathrm{V}_{3}$ decreased linearly with decreasing state 3 respiratory rate in the presence of $3.9 \mu \mathrm{M}$ LA. At a given $\mathrm{V}_{3}$, increasing LA decreased $\mathrm{V}_{\text {cyt cons }}$ (data not shown). At a given LA, PUMP activity contribution $\left(\mathrm{V}_{\text {PUMP }}\right)$ to $\mathrm{V}_{3}$ was constant when $V_{3}$ was decreased. At a given $\mathrm{V}_{3}$, increasing LA increased $\mathrm{V}_{\text {PUMP }}$ (data not shown).

Figure 7 shows the effect of AOX activity on the ADP/O ratio plotted against $1 / \mathrm{V}_{3}$ which is varied with n-butyl malonate. In the presence of BHAM, AOX is inactive and the $\mathrm{ADP} / \mathrm{O}$ ratio is constant. This constancy is the requirement for the validity of the ADP/ $\mathrm{O}$ method for this purpose. When AOX is activated (no BHAM), the ADP/O ratio decreases when $V_{3}$ increases.

The contribution of the cytochrome pathway $\left(\mathrm{V}_{\text {cyt }}\right)$ and of $\mathrm{AOX}\left(\mathrm{V}_{\text {alt }}\right)$ were calculated from the pair measurements of ADP/ $\mathrm{O}$ and $\mathrm{V}_{3}$ in the presence or absence of BHAM (Figure 8). A decrease in $\mathrm{V}_{3}$ was accompanied by a sharp decrease in $\mathrm{V}_{\text {alt }}$, whereas $\mathrm{V}_{\text {cyt }}$ remained almost constant and then decreased clearly with reduced $\mathrm{V}_{\text {alt }}$. $\mathrm{V}_{\text {alt }}$ reached zero when $\mathrm{V}_{3}$ was inhibited by $40 \%$.

Thus, the ADP/O method has facilitated the first examination of the steady-state kinetics of ATP synthase and PUMP when both are active on the one hand, and of the steady-state kinetics of the cytochrome pathway and AOX when both are active. Thus, this approach allows the description of the electron partitioning between AOX and the cytochrome pathway and the proton electrochemical gradient $\left(\Delta \widetilde{\mu} \mathrm{H}^{+}\right)$partitioning between ATP synthase and PUMP, when the steady-state rate of the quinone reducing pathway is decreased by decreasing succinate availability.

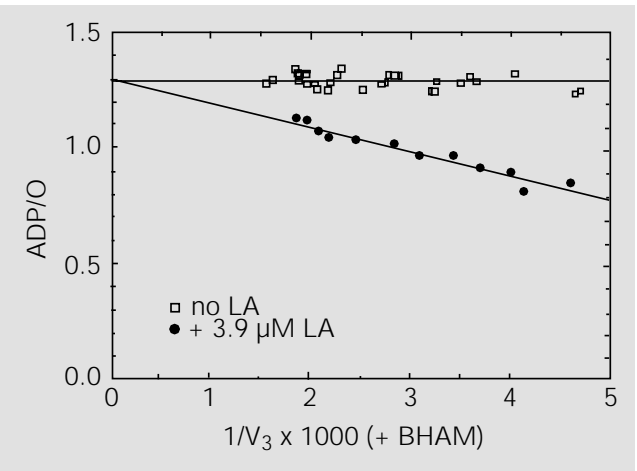

Figure 5 - Effect of $3.9 \mu \mathrm{M}$ linoleic acid (LA) on the ADP/O ratio plotted versus $1 / N_{3}$. ADP/O and $V_{3}$ (phosphorylating respiration) were measured with succinate plus rotenone as oxidizable substrate in the presence of $2 \mathrm{mM}$ benzohydroxamic acid (BHAM). $\mathrm{V}_{3}$ (expressed as $\mathrm{nmol} \mathrm{O} \mathrm{min}^{-1}$ mg protein $^{-1}$ ) was decreased by increasing concentrations of $n$ butyl malonate.

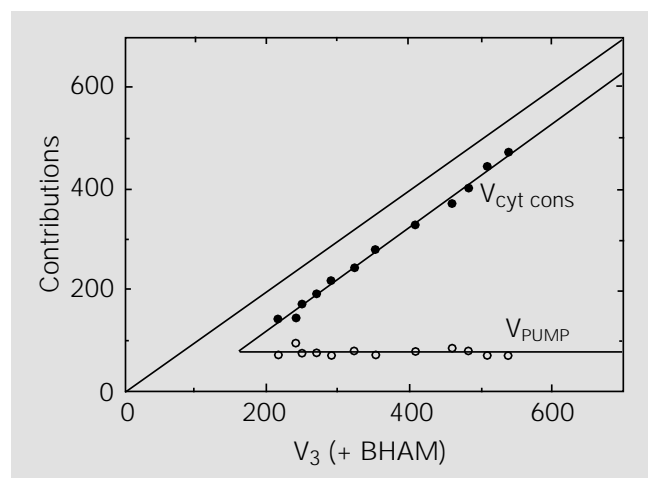

Figure 6 - Contributions of ATP synthesis and plant uncoupling mitochondrial protein (PUMP) activity in state 3 respiration. Contributions are calculated according to equations described in the text. $V_{\text {cyt cons }}$ is the ATP synthesis contribution, $V_{P U M P}$ is the PUMP activity contribution. Assay conditions and rate units as in Figure 5.

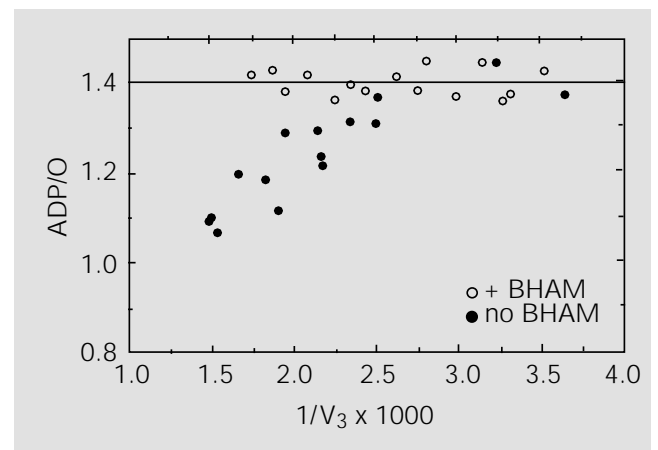

Figure 7 - Effect of AOX activity on the ADP/O ratio plotted versus $1 / N_{3}$. ADP/O and $V_{3}$ were measured with succinate plus rotenone as oxidizable substrate in the presence of $0.5 \%$ BSA and $1 \mathrm{mM}$ GTP, and in the presence or absence of $2 \mathrm{mM}$ benzohydroxamic acid (BHAM). $V_{3}$ (expressed as nmol O $\mathrm{min}^{-1} \mathrm{mg}$ protein $^{-1}$ ) was decreased by increasing concentrations of $n$-butyl malonate.

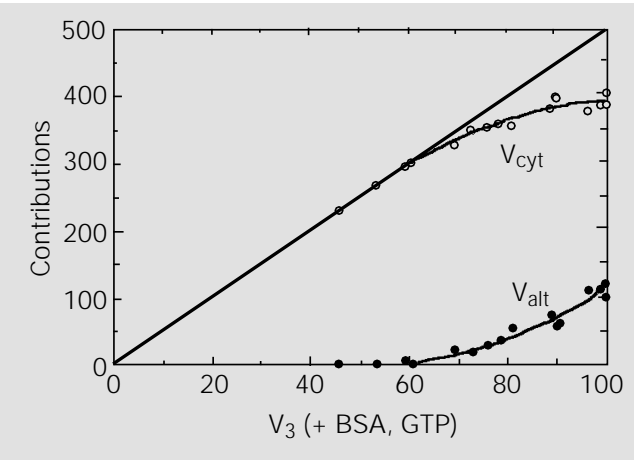

Figure 8 - Contributions of AOX activity and cytochrome pathway activity to state 3 respiration. Contributions are calculated according to equations described in the text. $V_{\text {alt }}$ is the altemative pathway contribution, and $V_{\text {cyt }}$ is the cytochrome pathway contribution. ADP/O and $\mathrm{V}_{3}$ are measured with succinate plus rotenone as oxidizable substrate in the presence of $0.5 \% \mathrm{BSA}$ and 1 mM GTP. $V_{3}$ (expressed as nmol $\mathrm{O} \mathrm{min}^{-1} \mathrm{mg}$ protein-1) was decreased by increasing concentrations of n-butyl malonate. 


\section{Extrapolation to general energy partitioning}

From these results it can be anticipated how the three pathways could contribute to overall state 3 respiration when LA concentration increases and when availability of an oxidizable substrate decreases, everything else being constant, namely enzyme concentrations and activators (Figure 9). In the absence of FFA (Figure 9, no LA) phosphorylating respiration $\left(\mathrm{V}_{3}\right)$ is the sum of the cytochrome pathway contribution $\left(\mathrm{V}_{\text {cyt }}\right)$ and of the AOX contribution $\left(\mathrm{V}_{\text {alt }}\right)$. When substrate availability decreases, $V_{\text {alt }}$ decreases faster compared to $\mathrm{V}_{\text {cyt }}$ and is equal to zero when $\mathrm{V}_{3}$ is inhibited by about $40 \%$. In the presence of low FFA concentrations (Figure 9, 3.9 $\mu \mathrm{M}$ LA), $V_{\text {alt }}$ is inhibited. $V_{3}$ is the sum of $V_{\text {alt }}$, of the ATP synthesis contribution $\left(\mathrm{V}_{\text {cyt cons }}\right)$ and of PUMP activity contribution $\left(\mathrm{V}_{\mathrm{PUMP}}\right)$. When substrate availability decreases $V_{\text {alt }}$ decreases faster than $\mathrm{V}_{\text {cyt cons }}$ while $\mathrm{V}_{\text {PUMP }}$

Figure 9 - Contributions of the three pathways ( $\mathrm{V}_{\text {cyt, }}, \mathrm{V}_{\mathrm{PUMP}}$, and $V_{\text {alt }}$ ) to overall state 3 respiration. For details see text.

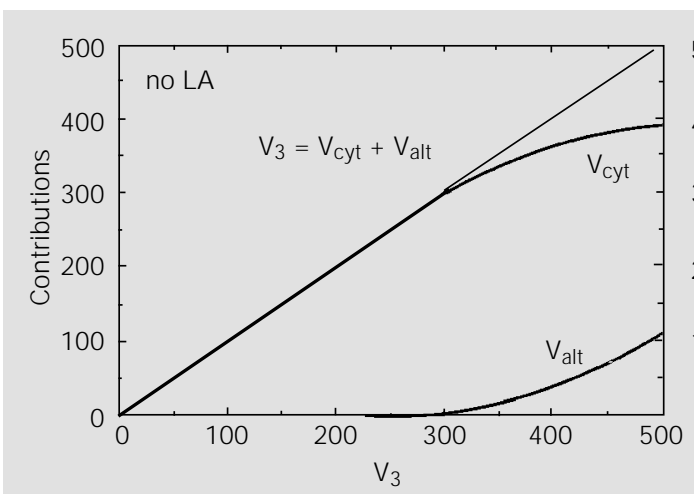

remains constant until $60 \%$ inhibition. When FFA concentration is around $15 \mu \mathrm{M}$ (Figure $9,15 \mu \mathrm{M} \mathrm{LA}), \mathrm{V}_{\text {alt }}$ is equal to zero and $\mathrm{V}_{3}$ is the sum of $\mathrm{V}_{\text {cyt cons }}$ and $\mathrm{V}_{\text {PUMP }}$ which remains constant until $\mathrm{V}_{\text {cyt cons }}$ is 0 due to the decrease in substrate availability. With high FFA concentrations (Figure 9, $>30 \mu \mathrm{M}$ LA), all the redox energy is dissipated into heat as $V_{\text {cyt }}$ cons is zero and $V_{3}$ is equal to $V_{P U M P}$.

\section{Conclusions}

Immunoblotting of mitochondrial proteins of green tomato mitochondria indicates that AOX and PUMP are present simultaneously in these mitochondria. The discovery of a functional connection between AOX and PUMP through a common regulation answers the puzzling question regarding their coexistence. Indeed, FFAs that activate PUMP drastically inhibit AOX. This observation leads to the proposal that AOX and PUMP never work simultaneously at their

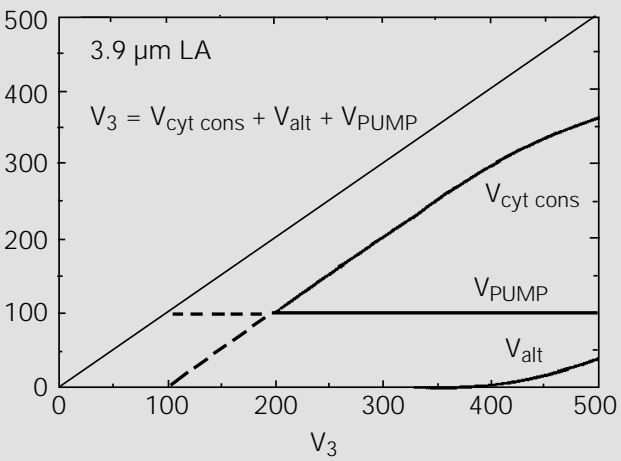

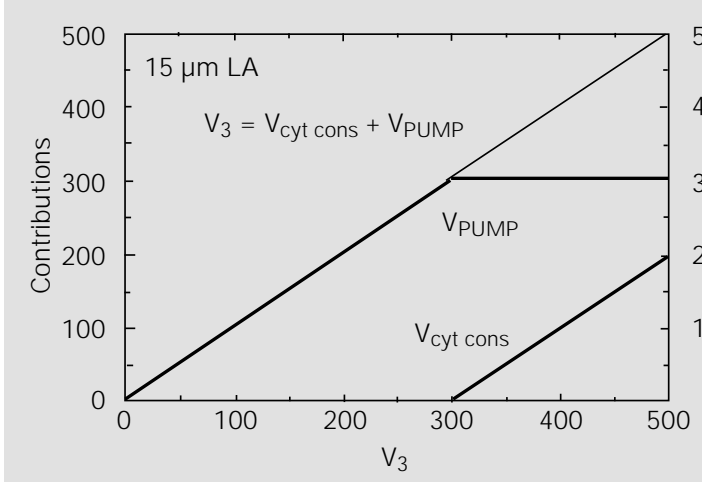

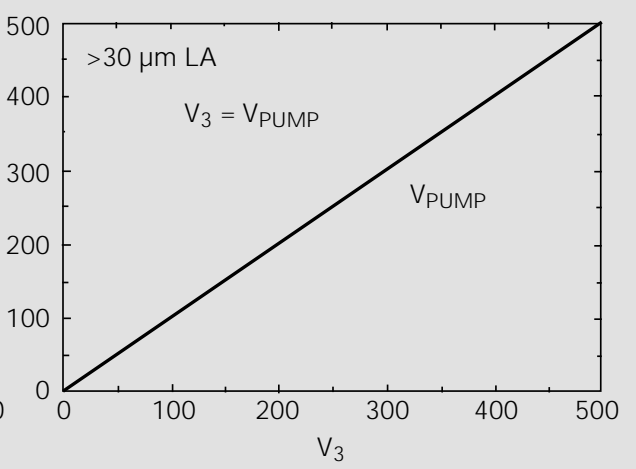


maximal activity and suggests that these two energy-dissipating enzymes could work sequentially. This proposal has been verified during post-harvest ripening of tomato fruit. Indeed, the early parallel decrease in AOX activity and protein expression does not follow the late parallel decrease in PUMP activity and protein expression. The $\mathrm{ADP} / \mathrm{O}$ method developed to determine the contribution of AOX and the cytochrome pathway to the overall state 3 respiration of Acanthamoeba castellanii (22) has permitted the description of the kinetics of the AOX and cytochrome pathway contributions when PUMP is inactive and the kinetics of $V_{\text {cyt cons }}$
(ATP synthase) and PUMP contributions when AOX is blocked. When state 3 respiration is decreased by substrate limitation, the contribution of AOX decreases sharply to zero (at $40 \%$ inhibition of $\mathrm{V}_{3}$ ), in contrast to the contribution of PUMP that remains constant until $60 \%$ inhibition. These last results support a functional additivity model for the three pathways in the overall state 3 respiration when FFA concentration increases.

\section{Acknowledgments}

We thank Dr. C. Sluse-Goffart for a critical reading of the manuscript.

\section{References}

1. Sluse FE \& J armuszkiewicz W (1998). Alternative oxidase in the branched mitochondrial respiratory network: an overview on structure, function, regulation, and role. Brazilian J ournal of Medical and Biological Research, 31: 733-747.

2. Vanlerberghe GC \& Mclntosh L (1997). Alternative oxidase: from gene to function. Annual Review of Plant Physiology and Molecular Biology, 48: 703-734.

3. Wagner AM \& Moore AL (1997). Structure and function of the plant alternative oxidase: its putative role in the oxygen defense mechanism. Bioscience Reports, 17: 319-333.

4. Vercesi AE, Martins IS, Silva MAP, Leite HMF, Cuccovia IM \& Chaimovich $\mathrm{H}$ (1995). PUM Ping plants. Nature, 375: 24.

5. Vercesi $A E$, Chaimovich $H \&$ Cuccovia IM (1997). A plant uncoupling mitochondrial protein, PUMP. Recent Research Development in Plant Physiology, 1: 85-91.

6. J ezek P, Costa ADT \& Vercesi AE (1997). Reconstituted plant uncoupling mitochondrial protein allows for proton translocation via fatty acid cycling mechanism. J ournal of Biological Chemistry, 272: 24272-24278.

7. J ezek $P$, Engstova $H$, Zackova $M$, Vercesi $A E$, Costa ADT, Arruda $P \&$ Garlid K (1998). Fatty acid cycling mechanism and mitochondrial uncoupling proteins. Biochimica et Biophysica Acta, 1365: 319327.

8. J armuszkiewicz W, Almeida AM, SluseGoffart CM, Sluse FE \& Vercesi AE (1998).
Linoleic acid-induced activity of plant uncoupling mitochondrial protein in purified tomato fruit mitochondria during resting, phosphorylating, and progressively uncoupled respiration. J ournal of Biological Chemistry, 273: 34882-34886.

9. Garlid KD, Orosz DE, Modriansky $M$, Vassanelli S \& J ezek P (1996). On the mechanism of fatty acid-induced proton transport by mitochondrial uncoupling protein. J ournal of Biological Chemistry, 269: 2615-2720.

10. Skulachev VP (1998). Uncoupling: new approaches to an old problem of bioenergetics. Biochimica et Biophysica Acta, 1363: 100-124.

11. Meeuse BJ D (1975). Thermogenic respiration in Aroids. Annual Review of Plant Physiology, 26: 117-126.

12. Day DA, Arron GP \& Laties GG (1980). Nature and control of respiratory pathways in plants: the interaction of cyanideresistant respiration with cyanide-sensitive pathway. In: Davies DD (Editor), Biochemistry of Plants. Vol. 4. Academic Press Inc., New York, 197-241.

13. Cruz-Hernandez A\& Gomez-Lim MA (1995). Alternative oxidase from mango (Mangifera indica, $\mathrm{L}$ ) is differentially regulated during fruit ripening. Planta, 197: 569-576.

14. Kumar S, Patil BC \& Sinha SK (1990). Cyanide-resistant respiration is involved in temperature rise in ripening mangoes. Biochemical and Biophysical Research Communications, 168: 818-822.
15. Kumar S \& Sinha SK (1992). Alternative respiration and heat production in ripening banana fruits (Musa paradisica var. Mysore Kadali ). J ournal of Experimental Botany, 43: 1639-1642.

16. Sluse FE, Almeida AM, J armuszkiewicz W \& Vercesi AE (1998). Free fatty acids regulate the uncoupling protein and alternative oxidase activities in plant mitochondria. FEBS Letters, 433: 237-240.

17. Kay CJ \& PalmerJ M (1985). Solubilization of the alternative oxidase of cuckoo-pint (Arum maculatum) mitochondria. Biochemical J ournal, 228: 309-318.

18. Minagawa N, Sakajo S \& Yoshimoto A (1992). Effects of unsaturated fatty acids on cyanide-resistant respiration of mitochondria isolated from Hansenula anomala. Bioscience, Biotechnology, and Biochemistry, 56: 1342-1343.

19. Güçlü J, Paulin A \& Soudain P (1989). Changes in polar lipids during ripening and senescence of cherry tomato (Lycopersicon esculentum): Relation to climacteric and ethylene increases. Plant Physiology, 77: 413-419.

20. Rouet-Mayer MA, Valentova O, SimondCôte E, Daussant J \& Thévenot C (1995). Critical analysis of phospholipid hydrolyzing activities in ripening tomato fruits. Study by spectrofluorimetry and high-performance liquid chromatography. Lipids, 30: 739-746.

21. Almeida AM, J armuszkiewicz W, Khomsi H, Arruda P, Vercesi AE \& Sluse FE (1999). Cyanide-resistant, ATP-synthesis-sus- 
tained, and uncoupling-protein-sustained respiration during postharvest ripening of tomato fruit. Plant Physiology, 119: 13231329.

22. J armuszkiewicz W, Sluse-Goffart CM, Hryniewiecka L, Michejda J \& Sluse FE (1998). Electron partitioning between the two branching quinol-oxidizing pathways in Acanthamoeba castellanii mitochondria during steady-state state 3 respiration. J ournal of Biological Chemistry, 273: 10174-10180.

23. Sluse $F E$, J armuszkiewicz W, Almeida $A M$, Vercesi $A E$, Hryniewiecka $L \&$ SluseGoffart CM (1998). Determination of actual contributions of energy-dissipating pathways in mitochondrial respiration: the ADP/O method. In: Moller IM, Gardestrom P, Glimelius K \& Glaser E (Editors), Plant Mitochondria: From Gene to Function. Backhuys Publishers, Leiden, The Netherlands, 519-523. 